\title{
Anthocyanins in chokeberry and purple maize attenuate diet-induced metabolic syndrome in rats
}

\begin{abstract}
Objective: Increased consumption of fruits and vegetables as functional foods leads to the reduction of signs of metabolic syndrome. The aim of this study was to measure and compare cardiovascular, liver, and metabolic parameters following chronic administration of the same dose of anthocyanins either from chokeberry (CB) or purple maize (PM) in rats with dietinduced metabolic syndrome. Methods: Male Wistar rats were fed a maize starch (C) or highcarbohydrate, high-fat diet $(\mathrm{H})$ and divided into six groups for $16 \mathrm{wk}$. The rats were fed $\mathrm{C}, \mathrm{C}$ with $\mathrm{CB}$ or PM for the last 8 wk (CCB or CPM), H, H with $\mathrm{CB}$ or PM for the last 8 wk (HCB or HPM); $\mathrm{CB}$ and $\mathrm{PM}$ rats received $\sim 8 \mathrm{mg}$ anthocyanins $/ \mathrm{kg}$ daily. The rats were monitored for changes in blood pressure, cardiovascular and hepatic structure and function, glucose tolerance, and adipose tissue mass. Results: HCB and HPM rats showed reduced visceral adiposity index, total body fat mass, and systolic blood pressure; improved glucose tolerance, liver, and cardiovascular structure and function; decreased plasma triacylglycerols and total cholesterol compared with $\mathrm{H}$ rats. Inflammatory cell infiltration was reduced in heart and liver. Conclusion: $\mathrm{CB}$ and $\mathrm{PM}$ interventions gave similar responses, suggesting that anthocyanins are the bioactive molecules in the attenuation or reversal of metabolic syndrome by prevention of inflammation-induced damage.
\end{abstract}

Keyword: Anthocyanins; Aronia melanocarpa; Chokeberry; Metabolic syndrome; Purple maize; Zea mays 